\title{
PUBLICAÇÕES NA ÁREA DE CATÁLISE ENVOLVENDO INSTITUIÇÕES BRASILEIRAS: UMA COMPARAÇÃO ENTRE OS PERIÓDICOS ESPECIALIZADOS E OS DA SBQ
}

Eduardo Nicolau dos Santos e Rochel Montero Lago*

Departamento de Química, Instituto de Ciências Exatas, Universidade Federal de Minas Gerais, CP 702, 31270-901,

Belo Horizonte - MG, Brasil

\begin{abstract}
PUBLICATIONS IN THE AREA OF CATALYSIS INVOLVING BRAZILIAN INSTITUTIONS: A COMPARISON BETWEEN SPECIALYZED AND SBQ JOURNALS. In this article it is made a preliminary analysis of the number and impact of Brazilian publications in the area of catalysis between 2004 and 2006 in major specialized international journals as well as in the SBQ publications, i.e. Journal of Brazilian Chemical Society (JBCS) and "Química Nova" (QN). A "Specific Impact Factor" (SIF) has been defined, which reflects the average number of citations of the papers involving catalysis in these journals. The SIF of JBCS papers in catalysis is increasing significantly in the last years, reaching even higher values than Brazilian papers in foreigner journals. The papers published in QN involving catalysis also have shown an important increase in their SFI over the last years.
\end{abstract}

Keywords: catalysis; publications; impact factor.

\section{INTRODUÇÃO}

A pesquisa em catálise é multidisciplinar e envolve diversas áreas, tais como materiais, cinética química, organometálicos, química de coordenação, química de sólidos e de superfícies, química supramolecular, modelagem e química teórica, nanotecnologia, biotecnologia etc. Sua aplicação na indústria química é bastante diversificada, sendo intensamente utilizada nos setores petroquímico, plásticos, controle ambiental, entre outros. Sua utilização na síntese orgânica de moléculas complexas tem crescido intensamente e, em consequiência, catalisadores ocupam um lugar de destaque nos setores de química fina e de fármacos. O catalisador é, em muitos casos, o ponto-chave na proteção intelectual de processos químicos. Apesar de ser uma área de pesquisa já consolidada, a importância da catálise se renova constantemente na indústria e na academia. Esse fato fica evidenciado pela concessão dos Prêmios Nobel em Química de 2001 e de 2005. O prêmio de 2001 foi concedido a Willian S. Kowles (Monsanto Company, St. Louis, Missouri/USA), Ryoji Noyori (Nagoya University, Chikusa, Nagoya/Japan) e K. Barry Sharpless (The Srcipps Research Institute, La Jolla, California/USA) por seus avanços e desenvolvimentos na síntese orgânica enantiosseletiva utilizando catalisadores quirais, que permitiram a síntese industrial de produtos homoquirais de alto valor agregado, tais como componentes de fragrâncias e fármacos. O Nobel em Química de 2005 foi atribuído ao francês Yves Chauvin e aos norte-americanos Robert H. Grubbs e Richard R. Schrock por suas contribuições para a metátese de olefinas, uma reação que emprega catalisadores homogêneos e heterogêneos de metais de transição. As descobertas desses pesquisadores tiveram um grande impacto na síntese orgânica, tanto na academia como no setor indústrial, pois possibilitaram rotas práticas e econômicas para a síntese de moléculas orgânicas complexas.

A área de catálise irá, sem dúvida, contribuir para os novos desafios do mundo moderno, tais como a obtenção de combustíveis limpos e de fontes renováveis, a síntese de produtos naturais, a obtenção de novos fármacos e defensivos agrícolas. A catálise tem contribuído significativamente em processos que visam o desenvolvimento sustentável e a proteção ambiental, dentro do conceito de Química Verde ("Green Chemistry"). Catalisadores com seletivi-

*e-mail: rochel@ufmg.br dades próximas a $100 \%$ serão cada vez mais importantes, pois aumentam a eficiência dos processos, eliminado a geração de contaminantes e sub-produtos. Neste contexto, o conhecimento íntimo, em nível molecular, do fenômeno catalítico será cada vez mais relevante para o melhor controle da reação.

\section{A COMUNIDADE DE CATÁLISE NO BRASIL}

Embora a pesquisa em catálise seja relativamente recente no Brasil, a área pode ser considerada consolidada. Atualmente existem vários grupos acadêmicos de pesquisa de nível internacional em uma distribuição geográfica relativamente diversificada no país. Um fator decisivo para a agremiação dos profissionais envolvidos na área foi a realização continuada do "Seminário Brasileiro de Catálise", um encontro bienal que se realiza desde 1981 e que teve seu nome alterado para "Congresso Brasileiro de Catálise" na sua $11^{\mathrm{a}}$ versão.

A Sociedade Brasileira de Química criou em 1995 a Divisão de Catálise com o objetivo de congregar os membros da sociedade com interesse diferenciado pela área. Essa Divisão organiza regularmente "workshops" sobre temas de interesse contemporâneo em que a catálise desempenha um papel fundamental. Os temas abordados até o momento foram: "Catálise no Setor de Energia" (30 RASBQ), "Catálise na Petroquímica" (29a RASBQ), "Catálise na Oleoquímica" (28ª RASBQ), "Química sol-gel: Catálise e Novos Materiais" (25 $\mathrm{a}$ RSBQ), "II Workshop em Catálise Homogênea" (23 RASBQ), "Workshop em Catálise Homogênea" (21 $1^{\text {a }}$ RASBQ). Entretanto, por razões históricas e pela proximidade intrínseca da catálise com o setor industrial, muito da pesquisa na área no país, sobretudo em catálise heterogênea, tem sido desenvolvida por engenheiros químicos, um público que normalmente não freqüenta as reuniões da SBQ.

Os profissionais que tradicionalmente freqüentavam o Congresso Brasileiro de Catálise julgaram que a área tinha massa crítica e pujança suficientes para ter a sua própria sociedade. Assim, em 1997 foi criada a "Sociedade Brasileira de Catálise" (SBCat), hoje consolidada e membro de várias associações internacionais de catálise (www.sbcat.org.br).

Outro aspecto relevante é que o Brasil se destaca na América Latina na produção de catalisadores em escala industrial. A Fábrica 
Carioca de Catalisadores é lider na produção de catalisadores para craqueamento de petróleo na América do Sul. Oxiteno, Umicore e Degussa são outros exemplos de empresas que produzem catalisadores em território nacional.

\section{A PESQUISA EM CATÁLISE NO BRASIL}

Para se ter um panorama relativamente atualizado das principais áreas de pesquisa em catálise no Brasil, utilizou-se como base os trabalhos apresentados no $13^{\circ}$ Congresso Brasileiro de Catálise e $3^{\circ}$ MercoCat (Foz do Iguaçu, 2005) onde foram apresentados 346 trabalhos. O levantamento foi qualitativo e não teve como objetivo uma análise estatística ou quantitativa da área. Os trabalhos foram classificados por áreas, sendo que o número absoluto de trabalhos por área e sua participação percentual podem ser vistos na Figura 1.

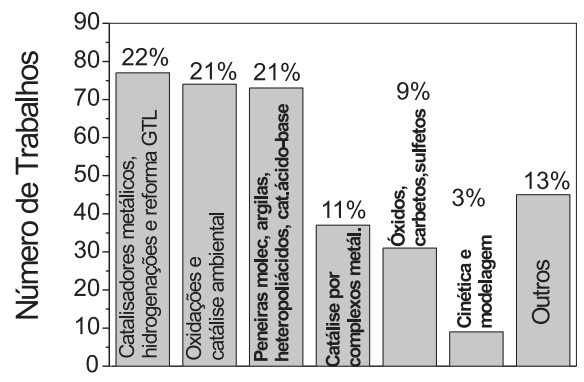

Áreas da Catálise

Figura 1. Número de trabalhos do $13^{\circ}$ Congresso Brasileiro de Catálise e do $3^{\circ}$ MercoCat dividido por áreas

Pode-se notar que três áreas se destacaram no referido congresso: "Catálise por metais" - metais como Pt, Pd, Ni, Ru, Rh, Co, Fe, $\mathrm{Re}, \mathrm{Sn}$ suportados em diferentes materiais compõem a maior parte dos trabalhos apresentados; "Oxidações e catálise ambiental" - aplicações ambientais da catálise, como fotocatálise, eliminação de NOx e SOx, tratamento de efluentes contribuem de forma expressiva; "Catálise ácido-base" - nesta área contribuem especialmente aplicações na área petroquímica. Ainda se destacam as áreas "Catálise por complexos metálicos" e "Catálise por óxidos, carbetos e sulfetos".

A Tabela 1 mostra alguns temas específicos que se destacaram nos trabalhos apresentados. Não foram explicitados os trabalhos em aplicações petroquímicas que, naturalmente, compõem uma importante parte dos trabalhos. A participação significativa de trabalhos na área ambiental e na conversão de metano segue uma tendência mundial observada em vários congressos internacionais na área de catálise, tais como o " $13^{\text {th }}$ International Congress on Catalysis", Paris - França, 2004; "Frontiers in Catalysis
Symposium", Vinsegrád- Hungria, 2005 e “ XX Congresso Iberoamericano de Catálise”, Gramado-Brasil, 2006. Observa-se ainda que algumas áreas específicas começam a despontar no Brasil, entre elas a conversão de óleos essenciais, a produção de biodiesel e reações envolvendo o uso de etanol, o que reflete a importância estratégica dessas áreas no Brasil.

\section{Periódicos científicos internacionais na área de catálise}

A área de catálise é privilegiada em termos de veículos de divulgação de resultados, tanto pelo número quanto pela qualidade dos periódicos disponíveis internacionalmente. Em uma busca no "Scifinder" ("Chemical Abstracts Service on-Line") foram encontrados 14 periódicos dedicados à área de catálise que tiveram publicações com participação de instituições brasileiras entre 2004 e 2006, conforme detalhado na Tabela 2.

Vale destacar que a maioria desses periódicos tem um fator de impacto superior a 2 e a classificação "Internacional A" pelo QUALIS da CAPES. Pode-se observar que, no período de 2004 a 2006, a porcentagem média da participação brasileira nos periódicos especializados em catálise analisados se aproxima de $2 \%$.

\section{Artigos da área de catálise publicados no JBCS}

Uma análise das áreas dos artigos envolvendo catálise publicados de 2004 a 2006 no JBCS pode ser vista na Figura 2.

Quando estes dados são comparados com os mostrados na Figura 1 (Congresso Brasileiro de Catálise), destaca-se uma diferença: no JBCS a maior parte dos artigos é da área de catálise por complexos de metais de transição ("catálise homogênea"). Essa diferença está provavelmente relacionada ao fato de que a catálise homogênea é desenvolvida principalmente por químicos e menos por engenheiros químicos, estando os últimos mais envolvidos com a "catálise heterogênea” (catálise por metais, óxidos metálicos, zeólitas etc...).

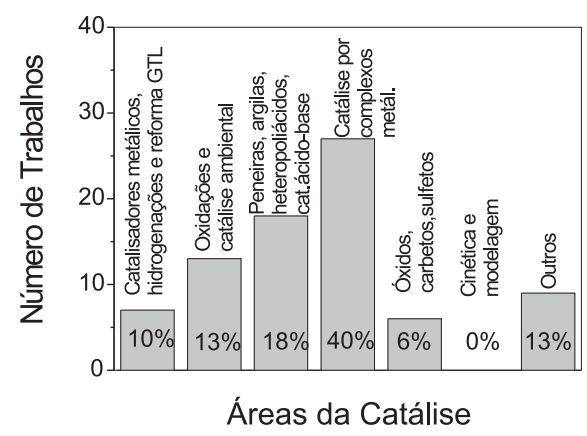

Figura 2. Número de trabalhos de catálise do JBCS entre 2004 e 2006 dividido por áreas

Tabela 1. Principais temas abordados nos trabalhos apresentados no evento conjunto $13^{\circ}$ Congresso Brasileiro de Catálise e $3^{\circ}$ MercoCat em Foz Iguaçu, 2005

\begin{tabular}{|c|c|}
\hline Tema & Destaques \\
\hline Catálise ambiental (ca. 14\% dos trabalhos) & $\begin{array}{l}\text { Fotocatálise (ca. 5\%); Oxidação de contaminates orgânicos voláteis (ca. } 4 \% \text { ); } \\
\text { Tratamento de efluentes aquosos (ca. } 3 \% \text { ); Destruição de NOx/SOx/fuligem } \\
\text { (ca. } 2 \% \text { ) }\end{array}$ \\
\hline Conversão de metano (ca. $8 \%$ dos trabalhos) & Importância devido às tecnologias GTL (“Gas to Liquid”) \\
\hline Química fina (ca. 8\% dos trabalhos) & Especialmente na transformação de óleos essenciais \\
\hline Transformações de etanol (ca. 5\% dos trabalhos) & Conversão de etanol em $\mathrm{H}_{2}$ e compostos de importância industrial \\
\hline Biodiesel e ácidos graxos (ca. 3\% dos trabalhos) & Produção do biodiesel no Brasil e transformação de ácidos graxos \\
\hline Pilha a combustível (ca. $2 \%$ dos trabalhos) & Importância do $\mathrm{H}_{2}$ como combustível \\
\hline
\end{tabular}


Tabela 2. Publicações em periódicos internacionais especializados na área de catálise envolvendo instituições brasileiras

\begin{tabular}{|c|c|c|c|c|c|}
\hline \multirow[t]{2}{*}{ Nome do periódico } & \multirow[t]{2}{*}{ Qualis $^{\mathrm{a}}$} & \multirow[t]{2}{*}{ Fator de impacto ${ }^{\mathrm{b}}$} & \multicolumn{3}{|c|}{ Número de publicações } \\
\hline & & & 2004 & 2005 & 2006 \\
\hline Advanced Synthesis \& Catalysis & IA & & 2 & 2 & 2 \\
\hline Applied Catalysis A: General & IA & 2.63 & 21 & 12 & 22 \\
\hline Applied Catal. B: Environmental & IA & 3.94 & 2 & 4 & 4 \\
\hline Catalysis Communications & IA & 1.06 & 8 & 4 & 6 \\
\hline Catalysis Letters & IA & 1.77 & 3 & 3 & 5 \\
\hline Catalysis Today & IA & 2.15 & 3 & 31 & 4 \\
\hline Journal of Catalysis & IA & 4.53 & 3 & 4 & 6 \\
\hline Journal Mol. Catal. A: Chemical & IA & 2.51 & 20 & 23 & 19 \\
\hline Journal Mol. Catal. B: Enzymatic & IB & 2.15 & 8 & 3 & 5 \\
\hline Kinetics and Catalysis & IC & 0.48 & - & - & 1 \\
\hline Progress in Catalysis Research & - & & - & 1 & - \\
\hline Reac. Kinetics and Catal. Letters & $\mathrm{IC}$ & 0.51 & 3 & 5 & 6 \\
\hline Studies Surf. Sci. and Catalysis & $\mathrm{IC}$ & & 23 & 9 & - \\
\hline Topics in Catalysis & - & 2.32 & 1 & - & - \\
\hline Total publicações brasileiras & & & 97 & 101 & 80 \\
\hline Total de publicações & & & 4899 & 4194 & 3966 \\
\hline Participação Brasileira (\%) & & & 1,98 & 2,41 & 2,02 \\
\hline
\end{tabular}

a Classificação dos periódicos pela CAPES para a área de Química em 2006; b Índice de avaliação do impacto científico de periódicos publicado pelo ISI em 2006; ${ }^{c}$ publicações envolvendo instituições brasileiras

Tabela 3. Análise dos artigos publicados no JBCS e na Química Nova na área de catálise

\begin{tabular}{|c|c|c|c|c|}
\hline Periódico & & 2004 & 2005 & 2006 \\
\hline \multirow[t]{4}{*}{ JBCS } & Número de artigos no JBCS envolvendo catálise & 17 em 153 & 30 em 223 & 33 em 235 \\
\hline & Expresso em \% com relação ao total de artigos & $11 \%$ & $13 \%$ & $14 \%$ \\
\hline & Fator de Impacto Específico para catálise ${ }^{\mathrm{a}}$ & 1,20 & 1,70 & 2,00 \\
\hline & Fator de Impacto Específico expurgando artigos ${ }^{\mathrm{b}}$ & 1,35 & 1,95 & 2,14 \\
\hline \multirow[t]{3}{*}{ QN } & Número de artigos na QN envolvendo catálise & 20 em 171 & 20 em 182 & 21 em 233 \\
\hline & Expresso em \% com relação ao total de artigos & $12 \%$ & $11 \%$ & $9 \%$ \\
\hline & Fator de Impacto Específico para catálise ${ }^{\mathrm{a}}$ & 0,40 & 0,47 & 0,85 \\
\hline \multirow[t]{2}{*}{$\begin{array}{l}\text { Periódicos estrangeiros } \\
\text { especializados em catálise }\end{array}$} & $\begin{array}{l}\text { Fator de Impacto Específico para artigos envolvendo } \\
\text { instituições brasileiras }\end{array}$ & 1,47 & 2,07 & 1,88 \\
\hline & $\begin{array}{l}\text { Número de publicações no JBCS relativo às } \\
\text { publicações em catálise em periódicos especializados }\end{array}$ & $17 \%$ & $30 \%$ & $41 \%$ \\
\hline
\end{tabular}

${ }^{\mathrm{a}}$ para metodologia, veja Nota $1 ;{ }^{\mathrm{b}}$ foram excluídos os artigos com baixo envolvimento com a área; ${ }^{\mathrm{c}}$ Nota 2

\section{Fator de Impacto Específico (FIE)}

Com a finalidade de avaliar o impacto dos artigos envolvendo catálise publicados no JBCS e na Química Nova, foi definido neste trabalho um indicador equivalente ao "Impact Factor", designado aqui como "Fator de Impacto Específico" (FIE), que pode, com as devidas adaptações, ser extrapolado para outras áreas. Para calculálo, utilizamos a seguinte equação:

$\mathrm{n}^{\text {o }}$ total de citações em 2006 de artigos em catálise de 2004 e 2005

FIE2006 $=\frac{\text { artigos em catálise de } 2004 \text { e } 2005}{\begin{array}{c}\mathrm{n}^{\circ} \text { total de artigos em } \\ \text { catálise de } 2004+2005\end{array}}$

Detalhes do cálculo do Fator de Impacto Específico (FEI) são comentados na Nota 1.

Um resumo dos resultados obtidos está apresentado na Tabela 3. É preciso salientar que, com o argumento de busca utilizado ("cataly*"), são localizados todos os artigos onde palavras como "catalysis", "catalyst(s)", "catalyzed" foram mencionadas no título, no resumo, nas palavras-chave e nas palavras-chave adicionais do artigo. Para JBCS e QN, em muitos exemplos, os artigos não tratavam de estudos catalíticos especificamente, mas apenas empregavam catalisadores em alguma etapa. Para efeito comparativo, o índice para o JBCS foi recalculado, expurgando-se manualmente os artigos cuja análise do resumo indicou pouco envolvimento com a área de catálise. Os resultados mostraram que o índice se elevou com esse procedimento (comparar na Tabela 3 as linhas 3 e 4)

É interessante observar que a porcentagem de publicações envolvendo de alguma maneira a área de catálise no JBCS e na QN, no período tratado, é elevada (9-14\%), considerando-se o número total de áreas envolvidas.

Comparando-se o Fator de Impacto Específico (FIE) do JBCS, expurgado ou não, com o FIE das publicações brasileiras nos jornais especializados de maior prestígio na área de catálise, pode-se observar que são bastante próximos. Estes dados indicam que o impacto e a qualidade das publicações do JBCS são próximas às publicações na área em periódicos estrangeiros especializados envolvendo instituições brasileiras. Isto sugere que o JBCS é um veículo adequado de publicações de qualidade na área de catálise. Pode-se também destacar que FIE para a área de catálise é superior ao "Impact Factor" do JBCS.

É interessante observar que as publicações de catálise no JBCS 
também têm crescido se comparadas com as publicações brasileiras em catálise em periódicos especializados estrangeiros. Em 2004 as publicações em catálise do JBCS correspondiam a $17 \%$ do número de publicações brasileiras em catálise em periódicos estrangeiros. Esse número cresce para 30\% em 2005 e para 41\% em 2006. Esses dados sugerem que o prestígio do JBCS junto aos profissionais brasileiros envolvidos em catálise tem aumentado.

A análise apresentada para o JBCS não é apropriada para avaliar a qualidade das publicações em QN, pois nesta, certamente, o idioma é um fator limitante para que os trabalhos ali publicados sejam citados mundialmente em uma proporção correspondente à sua qualidade e impacto.

Cabe destacar que os dados apresentados foram levantados de forma geral, sem a pretensão de uma quantificação rigorosa. O trabalho pretende apenas mostrar algumas tendências; estudos mais detalhados e específicos são necessários para eliminar as distorções que eventualmente ocorreram nesta análise.

\section{CONCLUSÃO}

Os dados analisados neste trabalho sugerem que as publicações na área de catálise no JBCS têm crescido de forma significativa com relação às publicações de outras áreas e também com relação às publicações brasileiras em periódicos estrangeiros especializados em catálise. O Fator de Impacto Específico sugere que a qualidade e o impacto das publicações em catálise no JBCS têm crescido e em 2006 chegou a ser maior que o FIE médio das publicações brasileiras em periódicos estrangeiros. Esses dados sugerem que JBCS é um veículo cada vez mais prestigiado para publicações na área de catálise.

\section{AGRADECIMENTOS}

Os autores agradecem aos Editores do JBCS e da QN, ao Prof. A. Pinto (UFRJ) pelas sugestões, à CAPES pela imensa contribuição à comunidade científica brasileira com Portal CAPES Periódicos.

\section{NOTAS}

Nota 1. (i) Busca no ISI Web of Knowledge (http://www.perio dicos.capes.gov.br/portugues/index.jsp), utilizando "General Search" com os seguintes delimitadores: $\mathrm{TS}=$ (palavras-chave características da área) AND $\mathrm{SO}=$ (nome do periódico) AND PY=(dois anos anteriores ao ano desejado); (ii) processar o "Citation Report"; (iii) dividir o número de citações do ano desejado pela somatória do número de artigos da área dos dois anos anteriores.

Para o caso específico deste trabalho, as entradas foram:

$\mathrm{TS}=($ cataly*) $\mathrm{AND} \mathrm{SO}=($ nome do periódico $)$ AND $\mathrm{PY}=($ dois anos anteriores ao ano desejado, exemplo: para o cálculo de 2006, utilizar intervalo 2004-2005).

Nota 2. Foi utilizada a metodologia descrita na nota 1 , utilizando $\mathrm{SO}=$ (APPLIED CATALYSIS A GENERAL OR APPLIED CATALYSIS B ENVIRONMENTAL OR CATALYSIS COMMUNICATIONS OR CATALYSIS LETTERS OR CATALYSIS TODAY OR JOURNAL OF CATALYSIS OR JOURNAL OF MOLECULAR CATALYSIS A CHEMICAL OR JOURNAL OF MOLECULAR CATALYSIS B ENZYMATIC) AND PY=(2004-2005) AND $\mathrm{AD}=(\mathrm{BRAZIL})$ 